\title{
The Human Mind: Animal Heritage and Cultural Vectors
}

\author{
Jorge A. Colombo, Md, Phd \\ Unidad Neurobiologia Aplicada (Cemic-Conicet), Buenos Aires, Argentina
}

\begin{abstract}
Humans are biological entities with social and cultural history; both dimensions metaphorically expressed as the "Biological-" and "Cultural-tectonic plates". In an evolutive context, the concept of predation applied to the Natural Kingdom appears as an anticipation to our species' cultural predatory behaviors. They include dynamic components associated with violence linked to social prevalence, reproductive behavior, territoriality, and access to food resources, and, in general, to aggressive or maladaptive behaviors. Another view of our "Cultural tectonic plate" has been lucidly expressed by Eduardo Colombo (2012): "the structure of our basic knowledge represents an epistemological field built on the old archetype of submission, where the subject is subdued by the networks that weave the practices and discourses that condition it; let's say to be brief, a subject determined by the structure of the system." Based on the behaviors of the chimpanzee and the bonobo, who share a common ancestor to Homo, Boehm (2012) poses that humans would keep behavioral styles from chimpanzees (tendency to conflict, male predominance), and bonobos (preventive behavior, female predominance). This anticipates a potential behavioral bipolarity with uneven prevalence distribution among individuals and social organizations. Based on social repression or "socialization", cultural strata of variable "thickness" have been constructed on top of drives implicit to our animal condition. Nevertheless, it failed in their deactivation, only in reformulating or repressing them.
\end{abstract}

To what extent our evolutionary inheritance is still conditioning our human social construction and individual behaviors? Profound cultural changes are only possible and enduring if we come to grips with our true primary condition.

Humans are biological entities with social and cultural history; both events metaphorically expressed as the "Biological-" and the "Cultural-tectonic plates" which synthesize basic interaction dynamics of our desires, frustrations, problems of adaptation, belligerence, and social sensitivity. 


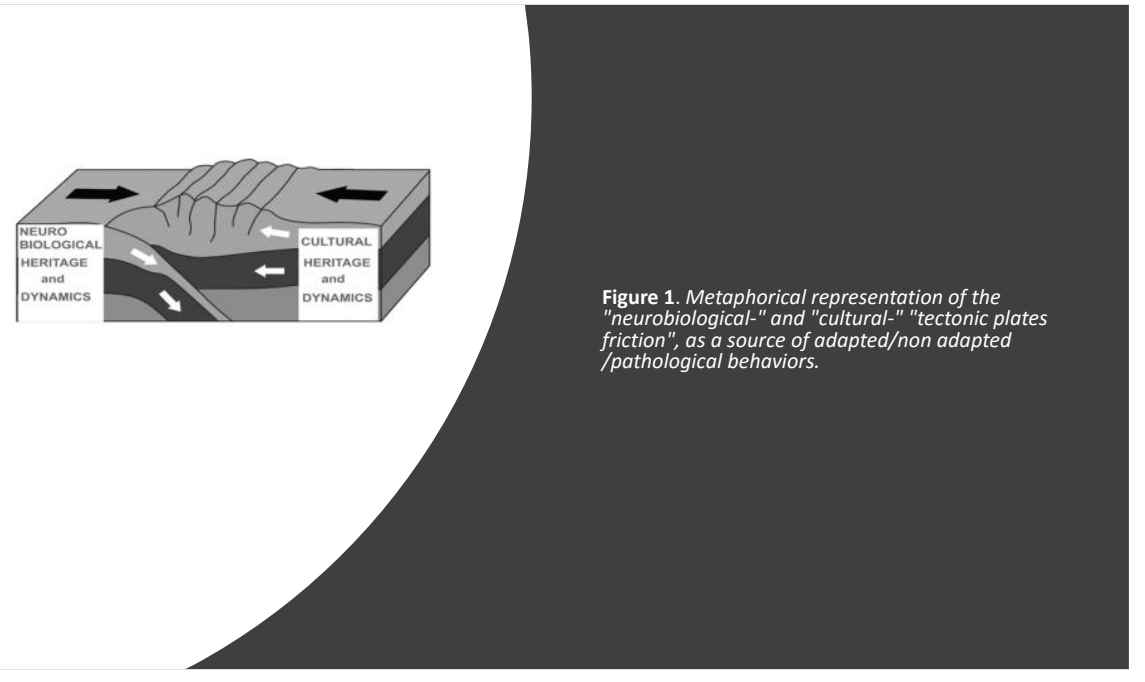

In this evolutive context, the concept of predation applied to the Natural Kingdom appears as an anticipation to our species' cultural predatory behaviors.

They include components associated with violence linked to social prevalence, reproductive behavior, territoriality, and access to food resources.

Another view of our "Cultural tectonic plate" has been lucidly expressed by Eduardo Colombo (2012):

“... the structure of our basic knowledge represents an epistemological field built on the old archetype of submission, where the subject is subdued by the networks that weave the practices and discourses that condition it; let's say to be brief, a subject determined by the structure of the system."

Cognitive processing involves distributed neural circuits as a substrate. The most disturbing from an intellectual point of view is that much of the former appears to be at the subconscious level. According to some authors, what appears at the conscious level, expressed temporarily at a specific time, are the events we can manipulate as a working memory in our executive behavior domain and corresponding to the explicit memory (Dietrich, 2015).

For comparison purposes, this would represent much less than the 10-15 percent that emerges from an iceberg on the surface (Dietrich, 2015) (Figure 2). 
20-22 March, 2020

Berlin, Germany $10^{\text {th }}$ International Conference on

Humanities, Psychology \& Social Sciences

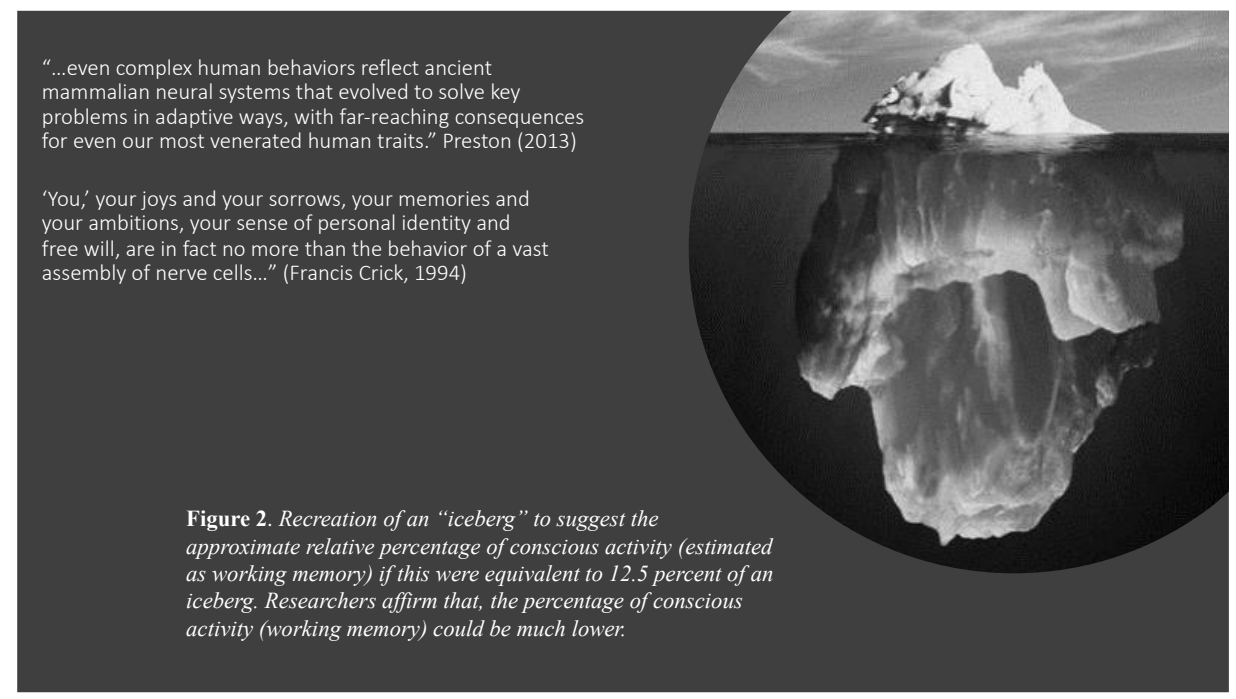

According to Thorstein Veblen (1899),

"The predatory phase of culture is attained only when the predatory attitude has become the habitual and accredited spiritual attitude for the members of the group...."

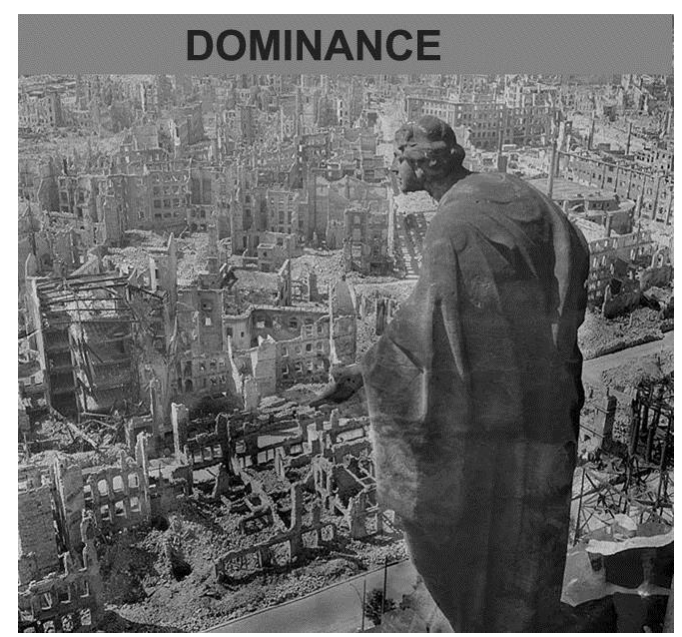

Figure 3. "With God on our side..." (Bob Dylan) (Modified from es.wikipedia.org.) "Dresden Destroyed. Scene from City Hall, 1945." The author is R. Peter.

Disparity of currents of scientific thought applied to evolutive issues has been used to support social and ideological movements involved with human rights, either as segregationist or inclusive. Within this context, it ought to be mentioned an event that had an impact on social anthropology toward the end of the XIX century and the construction of political thoughts at the beginning of the XX century. Such an event was produced in part by Broca's classification of cranial capacity in several human "races" (ethnic). It implied a clear underestimation of the relative cranial capacity of native American and African ethnicities, compared with those belonging to Europeans or Arians, and the implied cognitive performance. 
20-22 March, 2020

Berlin, Germany $10^{\text {th }}$ International Conference on

Humanities, Psychology \& Social Sciences

According to Boehm (2012), the evolutive predecessor - the ancestral Pan of hominid primates - to Homo, the chimpanzee (Pan troglodytes, or common chimpanzee) and the bonobo (Pan paniscus, or pygmy chimpanzee) would have lived in social constructions based on hierarchical dominance. Remarkably, there is a sharp difference in conflict management exerted by chimpanzees (tendency to conflict, male predominance), and bonobos (preventive behavior, female predominance).

Based on the behaviors of the chimpanzee and the bonobo, who share a common ancestor to Homo, Boehm (2012) poses that humans would keep behavioral styles from chimpanzees (tendency to conflict, male predominance), and bonobos (preventive behavior, female predominance). This anticipates a potential behavioral bipolarity with uneven prevalence distribution among individual characters and social organizations. Final behavioral phenotype would depend on socio-cultural and socio-biological conditions.

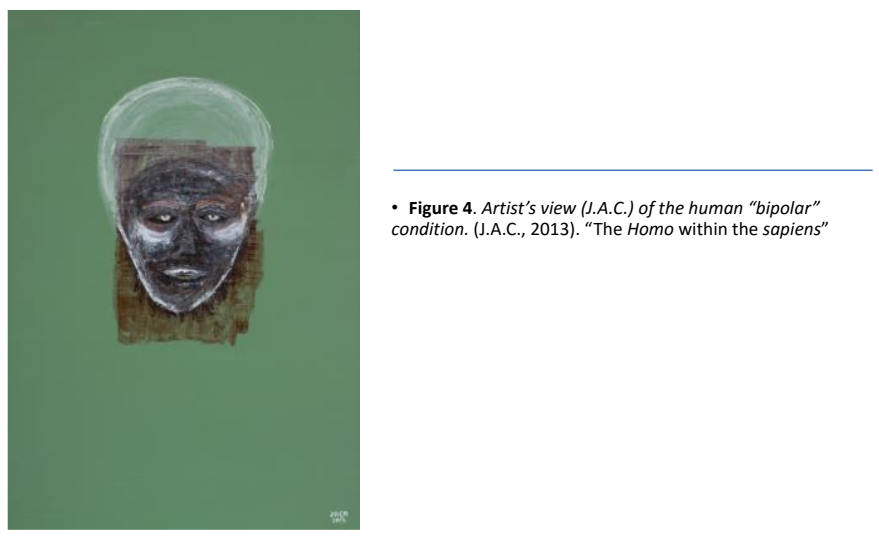

If we observe carefully, we can see that power, prevalence, territorial behavior and privileges also feed behaviors shared by other species, although we construct social norms and virtual agents aimed at buffering such circumstances. Primary forms of dealing with disputes involve either aggression, consensus, or submissiveness. Do they represent the spectrum of basic behaviors associated with species ancestral to $H$. sapiens and nonhuman primates? Gorillas and chimpanzees in a community express political actions aimed at obtaining sexual, feeding, or hierarchical privileges (De Waal, 2007).

As van Schaik (2016) states, "Biologically speaking, we are apes that are part of mammalian radiation, primates." "Humans are African great apes".

In conclusion, based on social repression or "socialization", cultural strata of variable "thickness" have been constructed on top of drives implicit to our animal condition. Nevertheless, it failed in their deactivation, only in reformulating or repressing them. Consequently, friction between these "geological plates" - of biological and cultural origin -, conditions various aggressive or maladaptive behaviors. In a context of social construction, such evolutive concepts of territory and neighbor to be suppressed, ought to be replaced by a cultural construction thriving toward cooperative behavior. 
20-22 March, 2020

Berlin, Germany $10^{\text {th }}$ International Conference on Humanities, Psychology \& Social Sciences

(Jorge A. Colombo “Our animal condition and social construction”, Science Publishers, 2019)

Key words: predatory behavior-socialization of animal drives-repression of animal drives-maladaptive behaviors-aggressiveness 